\title{
A light-addressable microfluidic device for label-free functional assays of bioengineered taste receptor cells via extracellular recording
}

\author{
Liping Du', Wei Chen ${ }^{1}$, Yulan Tian', Ping Zhu ${ }^{1}$, Jian Wang ${ }^{1}$, Wen Cai ${ }^{1}$, \\ Chunsheng $\mathrm{Wu}^{1 \times}$ \\ ${ }^{1}$ Institute of Medical Engineering, Department of Biophysics, School of Basic Medical Sciences, Health Science \\ Center, Xi'an Jiaotong University, Xi'an 710061, China
}

Received: 20 May 2018 / Accepted: 12 September 2018 / Published online: 20 April 2019

\begin{abstract}
The functional assay of chemical sensitive cells is of great importance for the preparation of sensitive elements towards the development of cell-based biosensors for chemical sensing. In this study, a novel light-addressable microfluidic device was developed by the integration of a light-addressable potentiometric sensor (LAPS) with a microfluidic chip for label-free functional assays of bioengineered taste receptor cells via extracellular recording. Extracellular potential changes of single bioengineered cells were recorded by LAPS. Microfluidic chip was capable of providing stable microenvironments for cell measurements with a well-defined concentration stimulus. Bioengineered taste receptor cells were utilized as a model of chemical sensitive cells and functional assayed by this light-addressable microfluidic device using bitter stimulation. The results indicate that this microfluidic device can efficiently monitor the membrane potential changes originated from bioengineered taste receptor cells in response to specific bitter stimulation. The bioengineered cells with responsive functions can be easily identified via the analysis on the firing rates of extracellular recording data. The expression of bitter receptors in the bioengineered taste receptor cells was further validated by the immunofluorescent staining results, which proved that the expression of specific bitter receptor was the main contribution to the responsive function of bioengineered cells. This microfluidic device can not only be used for the functional assays of chemical sensitive cells towards chemical sensing, but also suitable to be applied for the research on the chemical signal transduction mechanisms.
\end{abstract}

Keywords Cell-based biosensor, Light-addressable potentiometric sensor, Microfluidic device, Bioengineered taste receptor cell, Extracellular recording

\section{INTRODUCTION}

In recent decades, cell-based biosensors have attracted more and more attention due to their promising prospects and potential applications in many fields such as chemical sensing, toxin detection, and drug safety evaluation (Liu et al. 2014; Pancrazio et al. 1999).

Liping Du and Wei Chen have contributed equally.

$\bowtie$ Correspondence: wuchunsheng@xjtu.edu.cn (C. Wu)
Especially, taste receptor cells have been utilized as sensitive elements for the development of cell-based biosensors towards chemical sensing due to their unique capability of converting the specific environmental chemical signals to the cellular responses $(\mathrm{Wu}$ et al. 2014, 2017). Within the frame of cell-based biosensors, the key components are the sensitive cells that can not only respond to specific target analytes such as tastants, odorants, toxins, and drugs, but also generate responsive signals that can be detected by the transducers (Chandrashekar et al. 2006; Gilbertson 
et al. 2001; Matsunami et al. 2000). As a result, the preparation of sufficient functional cells is of great importance for the development of cell-based biosensors. It is thus highly desirable to develop novel approaches that can be employed for the functional assays of sensitive cells in a convenient and efficient manner.

Light-addressable potentiometric sensor (LAPS) has been reported suitable to be applied for the rapid and label-free functional assays of chemical receptors on the basis of extracellular recording from single cell (Du et al. 2014; Wu et al. 2009). LAPS belongs to field-effect devices based on the photoelectric effect inside the semiconductor (Hafeman et al. 1988; Owicki et al. 1994). LAPS with an electrolyte-insulatorsemiconductor structure allows for the detection of localized surface potential changes through the illumination of focused beam on the desired spot of LAPS chip (Ismail et al. 2003; Stein et al. 2004; Wang et al. 2018; $\mathrm{Xu}$ et al. 2005). Based on this configuration, measurement on the membrane potential changes from single cell cultured on the LAPS surface can be realized by the illumination of focused beam on the desirable single cell and monitor the fluctuations in photocurrent. LAPS provides a convenient and label-free approach for the function assays of chemical sensitive cells at the singlecell level. However, the complexity of large-volume measurement and the difficulties in controlling the injection speed and concentration of chemical stimulus in the detection chamber greatly limit the performance of the LAPS for functional cell assays.

With the fast advancement on the microfabrication techniques, microfluidic chip provides a new approach to overcome the limitations of LAPS measurement mentioned above (Bashir 2004; Richards Grayson et al. 2004; Wise and Najafi 1991). Microfluidic chip is able to process and control the trace liquid at the micro liter level (Dittrich and Manz 2006; Du et al. 2017; Duffy et al. 1998; Garstecki et al. 2006). In addition, the detection and other functional modules can be integrated on a single chip through the microchannel, pump, valve, mixer, and reactor structure, which has the advantages of small sample and reagent consumption, high reaction efficiency, and fast detection (Mark et al. 2010; Suzuki and Yoneyama 2003). Microfluidic chip provides a new technical platform for the research of single cell with regard to the fine control of the cellular microenvironment and cell culture conditions that are not available by traditional methods (Gao et al. 2004; Kimmerling et al. 2016; Mazutis et al. 2013). In addition, LAPS has been reported to be able to monitor the $\mathrm{pH}$ distribution inside the microfluidic channel (Miyamoto et al. 2014). Therefore, the combination of microfluidic chip with LAPS measurement setup provides a novel approach that could potentially overcome the limitations of traditional cell-based LAPS measurement.

This study developed a light-addressable microfluidic device for label-free functional assays of chemical sensitive cells by the integration of a LAPS chip with a microfluidic chip. This light-addressable microfluidic device combines the decisive advantage of microfluidic chip in the significant reduction of measurement volume, which could improve the stability of measurement system as well as the precision of desire concentrations of stimulus for cell assays. Bioengineered taste receptor cells that can respond to the specific bitter stimulations were used as a model of chemical sensitive cells and identified by this microfluidic device. In our experiment, the bioengineered taste receptor cells were cultured on the surface of LAPS chips. The responses of taste receptor cells to specific bitter stimulation were monitored via extracellular recording. Furthermore, responsive taste receptor cells can be efficiently identified via analysis on the firing rates of extracellular recording data. The expression of bitter receptors in the bioengineered taste receptor cells was further validated by the immunofluorescent staining experiments. It is worth to note that LAPS chips and bioengineered taste receptor cells used in this study are only for the demonstration of the technical feasibility of the novel approach for label-free functional assays of bioengineered cells.

\section{EXPERIMENTAL SECTION}

\section{Development of microfluidic device}

Microfluidic device was developed by the integration of LAPS chip with a commercial available microfluidic chip named $\mu$-Slide Chemotaxis 3D (ibidi GmbH, Martinsried, Germany). LAPS chip was fabricated on the substrate of p-type silicon wafer $(\langle 100\rangle, 10-15 \Omega \cdot \mathrm{cm})$. Briefly, a layer of $30-\mathrm{nm} \mathrm{SiO}_{2}$ was prepared on the surface of silicon wafer by thermal dry oxidization. Then, the rear side of the silicon wafer was grinded to remove the $\mathrm{SiO}_{2}$ layer and reduce the thickness of wafer to $100 \mu \mathrm{m}$. The upside of the wafer was deposited with a layer of $60-\mathrm{nm}$ $\mathrm{Si}_{3} \mathrm{~N}_{4}$ by plasma-enhanced chemical vapor deposition. To generate ohmic contact, a layer of $300-\mathrm{nm}$ Al was deposited on the rear side of the silicon wafer. The wafer was cut into square slices with sizes of $2 \mathrm{~cm} \times$ $2 \mathrm{~cm}$, which makes them suitable to be directly integrated with microfluidic chips.

In this study, $\mu$-Slide Chemotaxis 3D was used as a microfluidic chip to be integrated with LAPS chip. As 


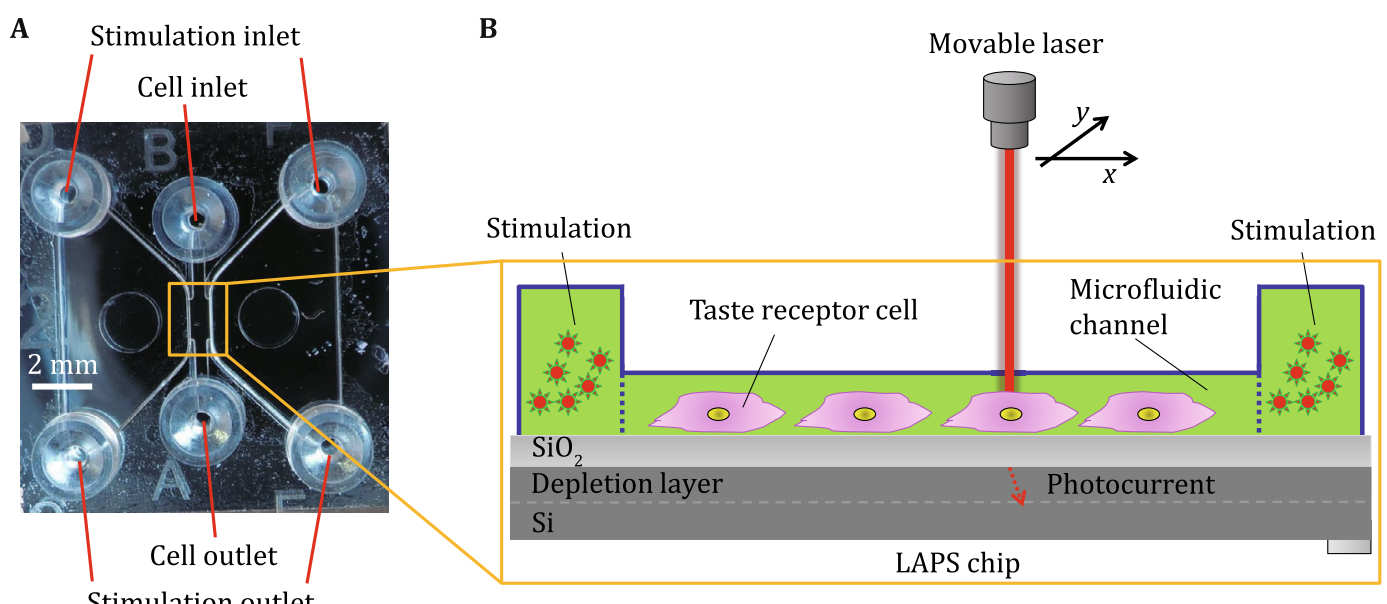

Fig. 1 A An image of a light-addressable microfluidic device, which shows the structure of microfluidic chip consisting of two stimulation chambers and one cell culture microchamber. B Schematic diagram showing the structure of the cell culture microchamber integrated with LAPS chip for the functional assays of single bioengineered taste receptor cells via extracellular recording of cell membrane potential changes

shown in Fig. 1A, $\mu$-Slide Chemotaxis 3D contains two large-volume reservoirs (volume of each reservoir is $60 \mu \mathrm{L}$ ) that are connected by a small gap. If each of the large reservoirs was filled with the same concentrations of chemical stimulations, a well-defined concentration inside this gap can be generated. The small gap with volume of $30 \mu \mathrm{L}$ was used for cell culture in which cells were exposed to a homogeneous concentration. Figure $1 \mathrm{~B}$ is the schematic diagram showing the cell culture microchamber integrated with LAPS chip.

\section{Preparation of bioengineered taste receptor cells}

Primary neuronal cells were prepared from the brain of Sprague-Dawley rats (1-3 day old). After decapitation, the rat brain was dissected and stored in cold Hanks balanced salt solution $\left(4{ }^{\circ} \mathrm{C}\right)$ free of calcium and magnesium (Gibco, USA). The brain was then incubated with the enzyme solution of $0.25 \%$ trypsin (pH 7.4-7.8) at $37{ }^{\circ} \mathrm{C}$ for $15 \mathrm{~min}$ and transferred to Dulbecco's modified Eagle's medium (DMEM) (Gibco, USA) with 10\% fetal bovine serum (FBS) for trituration with Pasteur pipet. The dissociated cells were then filtered with sieve of 200 holes/inch to obtain cell suspension. Then the cell suspension was maintained at $37{ }^{\circ} \mathrm{C}$ in DMEM with $10 \%$ FBS under standard conditions of humidified air with $5 \% \mathrm{CO}_{2}$. To prepare bioengineered taste receptor cells, rat primary neuronal cells were transfected with the plasmid pCEP4/rho-T2R4-His 6 as previous described (Wu et al. 2013), which allows for the expression of a taste receptor, T2R4, on the cellular membrane of primary neuronal cells.
For functional assays, the concentration of bioengineered taste receptor cells was adjusted to $3 \times 10^{6}$ cells $/ \mathrm{mL}$. Cells were loaded to the small gap of microfluidic device following the instruction of $\mu$-Slide Chemotaxis 3D manufacturer. Briefly, the filling ports of both large-volume reservoirs were closed with plugs, and then $6 \mu \mathrm{L}$ cell suspension was applied onto one of the filling ports of small gap. Immediately afterwards, the same pipet settings $(6 \mu \mathrm{L})$ were used to aspirate air from the opposite filling port to flush the cell suspension inside the entire small gap homogeneously. After the cell suspension reaches the pipet tip, both filling ports of the small gap were filled with liquid. Then, all plugs were gently removed from filling ports of both large-volume reservoirs and the slide with the cultivation lid was closed. The microfluidic device loaded with cells was incubated inside a sterile and humid atmosphere to minimize evaporation until the cells have attached. Chemical sensitive cells were attached to the LAPS surface after 2-h incubation. After cell attachment, non-adherent cells were removed by flushing the cell culture medium twice following the steps mentioned before. During measurement, both large-volume reservoirs were injected with chemical stimuli at desired concentration to stimulate the chemical sensitive cells.

For immunofluorescent staining experiments, bioengineered cells were incubated with primary antibody (anti-rho-tag rabbit IgG, diluted to $1: 50$ ) in $0.1 \mathrm{~mol} / \mathrm{L}$ PBS overnight at $4{ }^{\circ} \mathrm{C}$. Then, the bioengineered cells were washed with PBS $(\mathrm{pH}$ 7.4) for three times and incubated with secondary antibody (FITC-conjugated anti-rabbit IgG, diluted to $1: 100$ ) in $0.1 \mathrm{~mol} / \mathrm{L} \mathrm{PBS} \mathrm{(pH}$ 
7.3) for $2 \mathrm{~h}$ at room temperature. Bioengineered cells were then washed three times with PBS (pH 7.4) and fixed with $90 \%$ glycerin in PBS. Finally, cells were examined under a confocal fluorescence microscope (Leica TCS-SP, Germany).

\section{Measurement of chemical sensitive cells}

LAPS setup was employed to measure the responsive function of chemical sensitive cells to the specific chemical stimulations. As shown in Fig. 2, the configuration of LAPS setup mainly consists of a movable He-Ne semiconductor laser (Coherent Co., USA), a potentiostat (Model 273A, EG\&G Princeton Applied Research), a lock-in amplifier (model SR830 DSP, Stanford Research Systems), a 16-bit data collection card (National Instruments, USA), and a personal computer. To measure the function of single chemical sensitive cells, the microfluidic device cultured with cells was fixed under a microscope objective. The light (with wavelength of $543.5 \mathrm{~nm}$, modulation frequency at $4 \mathrm{kHz}$, and power of $5 \mathrm{~mW}$ ) generated by the semiconductor laser was focused to less than $10 \mu \mathrm{m}$ in diameter and move on the LAPS surface to illuminate the desired individual chemical sensitive cells for measurement. A mini $\mathrm{Ag} / \mathrm{AgCl}$ electrode and a Pt wire were used as reference electrode and counter electrode, respectively. Both of the reference electrode and counter electrode were connected to the electrolyte in the microchamber via the outlet of the microfluidic device during cell measurement. Single-cell membrane potential changes were monitored by recording the fluctuations in LAPS photocurrent, which were transmitted to the potentiostat via electrodes and amplified by the lock-in amplifier. The data were collected and stored in the personal computer via a 16-bit data collection card and the sampling rate of the photocurrent measurements was $40 \mathrm{kHz}$. The whole LAPS setup was controlled using customized LabVIEW software and shielded with a copper box. The temperature inside the copper box was controlled at $37 \pm 0.2{ }^{\circ} \mathrm{C}$.

\section{RESULTS AND DISCUSSION}

\section{Functional assays of bioengineered taste receptor cells}

Preparation of functional sensitive cells is of great importance for the development of cell-based biosensors. Especially, in case of utilization of chemical sensitive cells (e.g., taste receptor cells) as sensitive elements towards chemical sensing, it is highly desirable to perform functional assays on individual cells in order to identify cells that are capable of responding to the target chemical signals and converting these signals into cellular responses such as cell membrane potential changes. For this, a light-addressable microfluidic device was developed as shown in Fig. 1, in which a LAPS chip was integrated with a microfluidic chip. This lightaddressable microfluidic device provides an efficient platform for label-free and non-invasive functional assays of chemical sensitive cells at the single-cell level. The main advantages of this platform include the high stability of cellular measurement due to the microchamber for cell culture, well-controlled stimulus concentration, and single-cell recording and analysis benefited from the light addressability of LAPS.

To test the performance of this microfluidic device for the measurement of responsive function of chemical sensitive cells, a specific bitter receptor, T2R4, was expressed in rat neuronal cells, which exhibit properties
Fig. 2 Schematics of LAPS setup for the extracellular recording of cell membrane potential changes from single bioengineered taste receptor cells located in the cell culture microchamber of the lightaddressable microfluidic device

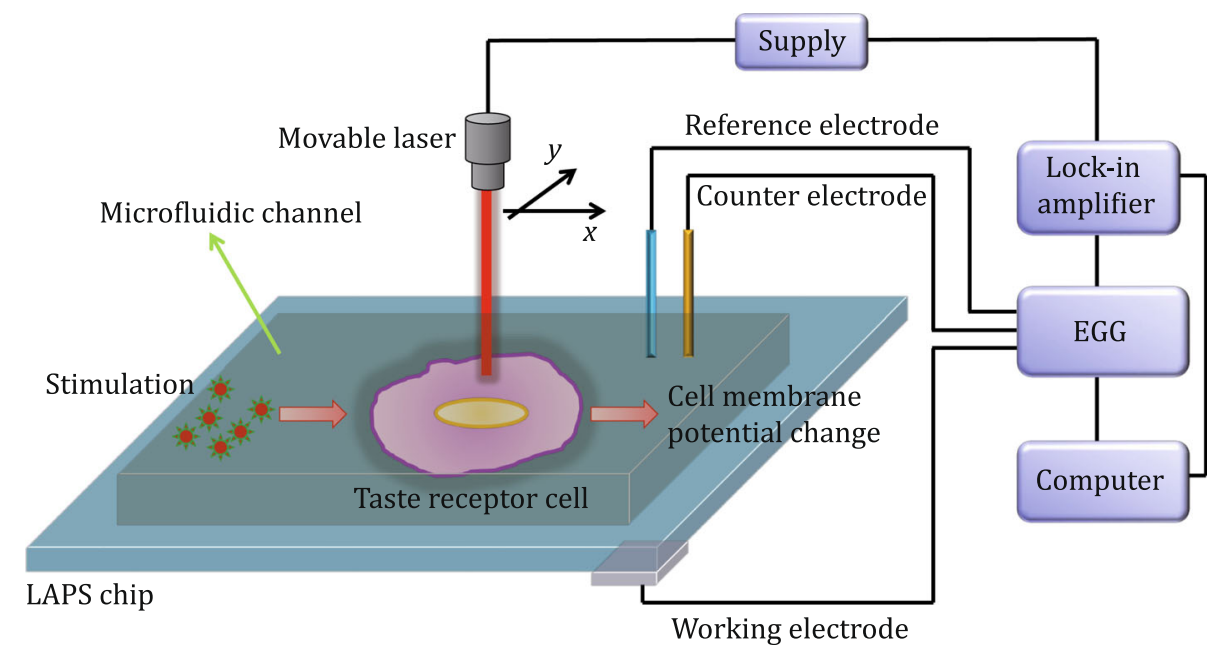


of neurons including the generation of responsive action potentials. The bioengineered taste receptor cells were cultured on the surface of LAPS chip via the microchamber of microfluidic device. LAPS, which is a surface-potential sensitive semiconductor device, was utilized to monitor single-cell membrane potential changes by illuminating the focused laser on the desired single target cell. To assay the chemical sensitive function of cells, specific chemical stimuli were applied to the cells via the two large-volume reservoirs of the microfluidic device. The responses of cells to the natural target ligands of T2R4, denatonium, were measured by recording the photocurrent fluctuations originated from cellular membrane potential changes. Figure $3 \mathrm{~A}$ shows the typical LAPS extracellular recordings from single bioengineered cells (without responsive function) under stimulation of $1 \mathrm{mmol} / \mathrm{L}$ denatonium, in which some firing spikes can be observed. This is mainly due to the lack of bitter receptor expression in rat neuronal cells. It is probably the indication of spontaneous action potentials, which can generate changes in cell membrane potentials. These potential changes can thus be readout by the extracellular recording of LAPS chip. Figure 3B shows the typical LAPS extracellular recordings from single bioengineered cells (with responsive function) under stimulation of $1 \mathrm{mmol} / \mathrm{L}$ denatonium, which shows much higher firing spike rate than that of bioengineered cells (without responsive function). This is mainly attributed to the expression of bitter receptor
(T2R4) in the bioengineered cells, which can drastically increase the responses of bioengineered cells to the specific bitter stimulation (denatonium). To further compare the differences in the firing rates between bioengineered cells with and without responsive function, we performed 42 extracellular recording measurements. For each measurement, the firing rate was calculated in which three times of the average noise level was set as the threshold for the extraction of number of the firing spikes within a certain time. In this study, the average noise level of extracellular recording was calculated to be $20 \mu \mathrm{V}$. As a result, the threshold for the extraction of firing spikes was set at $60 \mu \mathrm{V}$. Figure 3C shows statistical results of the firing rates recorded from control cells and bioengineered cells under bitter stimulation. It is indicated that the firing rates of responsive bioengineered cells (19.2 \pm $3.10 \mathrm{~Hz}$ ) is significantly higher than that of nonresponsive bioengineered cells $(5.6 \pm 1.30 \mathrm{~Hz}, P=$ $6.15 \times 10^{-5}, n=42$ ). By the comparison of extracellular recording signals and the calculation of firing rates, it is able to identify responsive bioengineered cells $(15$ cells) from non-responsive bioengineered cells (27 cells). All the results proves that this light-addressable microfluidic device can identify the responsive bioengineered taste cells by extracellular recording, where the responsive bioengineered cells show much higher firing rates than that of non-responsive bioengineered cells. In addition, the results demonstrate that this

A Non-responsive bioengineered cell

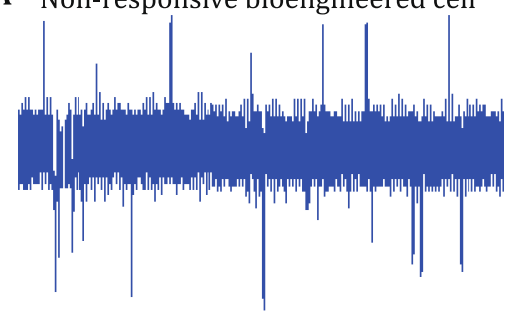

B Responsive bioengineered cell

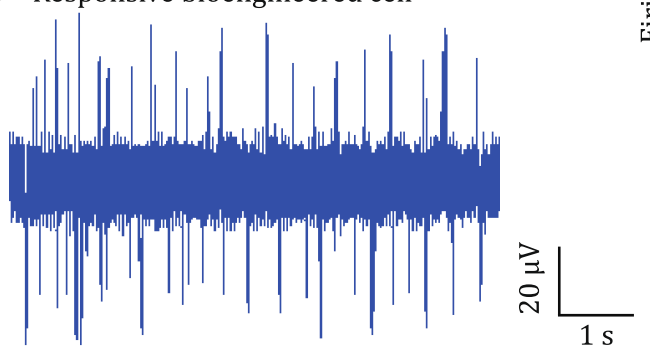

C

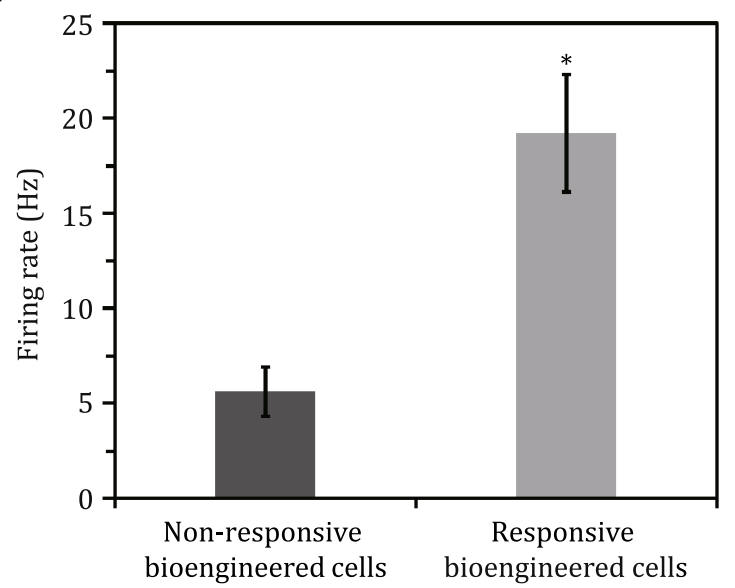

Fig. 3 A Typical LAPS extracellular recordings from single non-responsive bioengineered cell under stimulation of $1 \mathrm{mmol} / \mathrm{L}$ denatonium. B Typical LAPS extracellular recordings from single responsive bioengineered cell under stimulation of 1 mmol/L denatonium. C Statistical results of the firing rates recorded from non-responsive bioengineered cells and responsive bioengineered cells under stimulation of $1 \mathrm{mmol} / \mathrm{L}$ denatonium. All data are represented by the mean \pm SEM. ${ }^{*} P<0.01$, Student's $t$ test, $n=42$ 
microfluidic device-based biosensor can monitor the cell membrane potential changes resulting from the taste signal transduction mediated by bioengineered taste receptor cells. It can be used to study the special properties of taste receptors. However, more experiments are certainly desirable for the further application of this biosensor in practical.

\section{Expression of bitter receptor}

In this study, bioengineered cells prepared from rat primary neuronal cells were used as a model of chemical sensitive cells and expressed with a specific bitter receptor fused with a rho-tag. To validate the expression of bitter receptors in the bioengineered cells, immunofluorescent staining experiments were performed with the help of antibodies that can recognize the rho-tag fused bitter receptors. Figure 4 shows the results of immunofluorescent staining, from which the cell morphology and sub-cellular distribution of fluorescence can be observed. On the one hand, the morphological results show that bioengineered cells grow well and show elongated and slender shapes, which indicate the maintaining of their native neuronal morphology to some extent. It is suggested that the neuronal cells are able to maintain their native structures and functions to some extent including the generation of action potentials. On the other hand, the sub-cellular distribution of fluorescent indicates the location of expressed bitter receptors. The fluorescent staining results show that around 1/3 bioengineered cells are expressed with bitter receptors. The expression efficiency is consistent to the results of extracellular recording, in which 15 cells were identified as responsive bioengineered cells among 42 bioengineered cells tested altogether. In addition, the expressed bitter receptors are mainly located on the plasma membrane of neuronal cells, which can facilitate the specific
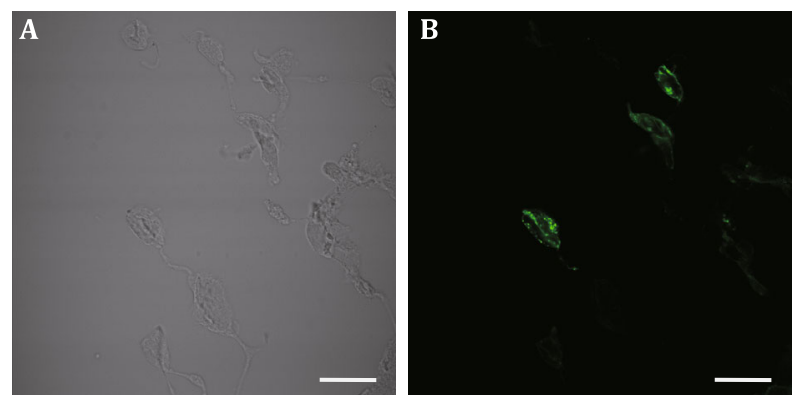

Fig. 4 Immunofluorescent staining images of bioengineered taste receptor cells prepared from rat primary neuronal cells transfected with pCEP4/rho-T2R4-His 6 taken under optical field (A) and fluorescent field (B). Scale bar: $20 \mu \mathrm{m}$ interactions between bitter receptors and bitter compounds. All the results prove that the expression of specific bitter receptor in the bioengineered rat neuronal cells was at an efficient manner, which contributed greatly to the responsive function of bioengineered cells. With minor modifications (e.g., changes in the types of expressed receptors), this light-addressable microfluidic device can be applied to the functional assays of other types of bioengineered receptor cells, and provides a novel approach for the preparation of sufficient functional cells suitable to be use as sensitive elements. Cellbased assays using this microfluidic device are also applicable to the research of signal transduction mechanisms of different types of membrane receptors such as olfactory receptors.

\section{CONCLUSION}

In this study, a light-addressable microfluidic device was developed for label-free functional assays of bioengineered cells via extracellular recording, which is crucial for the development of cell-based biosensors. This lightaddressable microfluidic device was successfully applied in the identification of responsive bioengineered taste receptor cells prepared from rat primary neuronal cells transfected with plasmid containing the cDNA of a specific bitter receptor. This microfluidic device provides high-stable and high-efficient performances in functional cell assays towards identification of responsive bioengineered taste receptor cells. In addition, the immunofluorescent staining results matched well with the extracellular recording data, which proved the expression of specific bitter receptors is the main contribution to the responsive function of bioengineered taste receptor cells. It is suggested that this microfluidic device can also be used for the functional assays of other chemical sensitive cells similar to bioengineered taste receptor cells such as olfactory cells. With further development, this microfluidic device may be also suitable for the research on the chemical signal transduction mechanisms at a single-cell level.

Acknowledgements This work was financially supported in part by Grants from the National Natural Science Foundation of China (31470956, 31661143030, 31700859, and 51861145307), the Doctoral Fund of Education Ministry of China (2016M602832), and the Fundamental Research Funds for the Central Universities, China.

\section{Compliance with Ethical Standards}

Conflict of interest Liping Du, Wei Chen, Yulan Tian, Ping Zhu, Jian Wang, Wen Cai, and Chunsheng Wu declare that they have no conflict of interest. 
Animal rights and informed consent All institutional and national guidelines for the care and use of laboratory animals were followed.

Open Access This article is distributed under the terms of the Creative Commons Attribution 4.0 International License (http:// creativecommons.org/licenses/by/4.0/), which permits unrestricted use, distribution, and reproduction in any medium, provided you give appropriate credit to the original author(s) and the source, provide a link to the Creative Commons license, and indicate if changes were made.

\section{References}

Bashir R (2004) BioMEMS: state-of-the-art in detection, opportunities and prospects. Adv Drug Deliv Rev 56:1565-1586

Chandrashekar J, Hoon MA, Ryba NJ, Zuker CS (2006) The receptors and cells for mammalian taste. Nature 444:288-294

Dittrich PS, Manz A (2006) Lab-on-a-chip: microfluidics in drug discovery. Nat Rev Drug Discovery 5:210-218

Du LP, Zou L, Zhao LH, Huang LQ Wang P, Wu CS (2014) Label-free functional assays of chemical receptors using a bioengineered cell-based biosensor with localized extracellular acidification measurement. Biosens Bioelectron 54:623-627

Du WB, Fang Q He QH, Fang ZL (2017) High-throughput nanoliter sample introduction microfluidic chip-based flow injection analysis system with gravity-driven flows. Anal Chem 77:1330-1337

Duffy DC, McDonald JC, Schueller OJ, Whitesides GM (1998) Rapid prototyping of microfluidic systems in poly(dimethylsiloxane). Anal Chem 70:4974-4984

Gao J, Yin XF, Fang ZL (2004) Integration of single cell injection, cell lysis, separation and detection of intracellular constituents on a microfluidic chip. Lab Chip 4:47-52

Garstecki P, Fuerstman MJ, Stone HA, Whitesides GM (2006) Formation of droplets and bubbles in a microfluidic T-junction-scaling and mechanism of break-up. Lab Chip 6:437-446

Gilbertson TA, Boughter JD, Zhang H, Smith DV (2001) Distribution of gustatory sensitivities in rat taste cells: whole-cell responses to apical chemical stimulation. J Neurosci 21:4931-4941

Hafeman DG, Parce JW, McConnell HM (1988) Light-addressable potentiometric sensor for biochemical systems. Science 240:1182-1185

Ismail $A B$, Yoshinobu T, Iwasaki $H$, Sugihara $H$, Yukimasa T, Hirata I, Iwata $H$ (2003) Investigation on light-addressable potentiometric sensor as a possible cell-semiconductor hybrid. Biosens Bioelectron 18:1509-1514

Kimmerling RJ, Lee Szeto G, Li JW, Genshaft AS, Kazer SW, Payer KR, de Riba Borrajo J, Blainey PC, Irvine DJ, Shalek AK, Manalis SR (2016) A microfluidic platform enabling singlecell RNA-seq of multigenerational lineages. Nat Commun $7: 10220$
Liu QJ, Wu CS, Cai H, Hu N, Zhou J, Wang P (2014) Cell-based biosensors and their application in biomedicine. Chem Rev 114:6423-6461

Mark D, Haeberle S, Roth G, von Stetten F, Zengerle R (2010) Microfluidic lab-on-a-chip platforms: requirements, characteristics and applications. Chem Soc Rev 39:1153-1182

Matsunami H, Montmayeur JP, Buck LB (2000) A family of candidate taste receptors in human and mouse. Nature 404:601-604

Mazutis L, Gilbert J, Ung WL, Weitz DA, Griffiths AD, Heyman JA (2013) Single-cell analysis and sorting using droplet-based microfluidics. Nat Protoc 8:870-891

Miyamoto K, Itabashi A, Wagner T, Schöning MJ, Yoshinobu T (2014) High-speed chemical imaging inside a microfluidic channel. Sens Actuators B 194:521-527

Owicki JC, Bousse LG, Hafeman DG, Kirk GL, Olson JD, Wada HG, Parce JW (1994) The light-addressable potentiometric sensor: principles and biological applications. Annu Rev Biophys Biomol Struct 23:87-113

Pancrazio JJ, Whelan JP, Borkholder DA, Ma W, Stenger DA (1999) Development, application of cell-based biosensors. Ann Biomed Eng 27:697-711

Richards Grayson AC, Shawgo RS, Johnson AM, Flynn NT, Li YW, Cima MJ, Langer R (2004) A bioMEMS review: MEMS technology for physiologically integrated devices. Proc IEEE 92:6-21

Stein B, George M, Gaub HE, Parak WJ (2004) Extracellular measurement of averaged ionic currents with the lightaddressable potentiometric sensor (LAPS). Sens Actuators B 98:299-304

Suzuki H, Yoneyama R (2003) Integrated microfluidic system with electrochemically actuated on-chip pumps and valves. Sens Actuators B 96:38-45

Wang J, Du LP, Krause S, Wu CS, Wang P (2018) Surface modification and construction of LAPS towards biosensing applications. Sens Actuators B 265:161-173

Wise KD, Najafi K (1991) Microfabrication techniques for integrated sensors and microsystems. Science 254:1335-1342

Wu CS, Chen P, Yu H, Liu QJ, Zong XL, Cai H, Wang P (2009) A novel biomimetic olfactory-based biosensor for single olfactory sensory neuron monitoring. Biosens Bioelectron 24:1498-1502

Wu CS, Du LP, Zou L, Huang LQ Wang P (2013) A biomimetic bitter receptor-based biosensor with high efficiency immobilization and purification using self-assembled aptamers. Analyst 138:5989-5994

Wu CS, Du LP, Zou L, Zhao LH, Huang LQ Wang P (2014) Recent advances in taste cell- and receptor-based biosensors. Sens Actuators B 201:75-85

Wu CS, Du Y-W, Huang LQ Galeczki YB, Dagan-Wiener A, Naim M, Niv M, Wang P (2017) Biomimetic sensors for the senses: towards better understanding of taste and odor sensation. Sensors 17:2881

Xu GX, Ye XS, Qin LF, Xu Y, Li Y, Li R, Wang P (2005) Cell-based biosensors based on light-addressable potentiometric sensors for single cell monitoring. Biosens Bioelectron 20:1757-1763 